\title{
Laparoscopic sleeve gastrectomy performed in a morbidly obese patient with gastrointestinal stromal tumor: a case report and literature review
}

Kenkichi Hashimoto ${ }^{*}$ (D), Yoshihisa Sakaguchi, Sho Nambara, Kensuke Kudou, Eiji Kusumoto, Keiji Yoshinaga, Tetsuya Kusumoto and Koji Ikejiri

\begin{abstract}
Background: Gastrointestinal stromal tumor (GIST) is the most frequent submucosal tumor, and with advancements of diagnostic modalities, the incidence of GIST cases diagnosed have increased. Similarly, prevalence of morbid obesity has also rapidly increased over the past decade. Notably, the incidence of GIST in obese patients was reported to be more frequent as compared to the general population. Despite local resection being the first choice for GIST treatment, extensive surgery should also be considered depending on the tumor size and location. Laparoscopic sleeve gastrectomy (LSG), the most popular bariatric procedure, could also be a concomitant treatment option for both morbid obesity and GIST when the tumor is contained within LSG the excision range. There are, however, few reports about LSG planned for GIST preoperatively.

Case presentation: A morbidly obese 46-year-old Japanese male (body weight of $105.4 \mathrm{~kg}$, body mass index (BMI) of $36.6 \mathrm{~kg} / \mathrm{m}^{2}$ ) was diagnosed with an intramural GIST in the gastric fundus. Because of his extreme visceral fat dominated obesity (visceral fat area of $386 \mathrm{~cm}^{2}$ ), in addition to the size and location of the tumor, we determined that it would be difficult to perform local resection. We planned LSG as a concomitant treatment for both GIST and morbid obesity. After the preoperative examination and 6 months of weight control, the patient lost enough weight to undergo LSG safely. Keeping enough distance away from the tumor, which we observed with an endoscope, we performed LSG to successfully resect the tumor. The patient was discharged uneventfully. Weight loss was successful as his BMI was $21.0 \mathrm{~kg} / \mathrm{m}^{2}$ at 3 months post-surgery.

Conclusion: We successfully performed LSG in a morbidly obese patient with a large GIST. This is the largest GIST concomitantly resected with LSG reported within current literature.
\end{abstract}

Keywords: GIST, Morbid obesity, Laparoscopic sleeve gastrectomy

\section{Background}

Morbid obesity has rapidly increased over the past decade. Bariatric surgery is the most effective therapy to treat obesity and its associated comorbidities. Although laparoscopic sleeve gastrectomy (LSG) is a relatively new

\footnotetext{
* Correspondence: kenkichi88@mac.com

Department of Gastroenterological Surgery/Clinical Research Institute,

National Kyushu Medical Center, 1-8-1 Jigyohama, Chuo-ku, Fukuoka, Japan
}

bariatric procedure, it overtook the incidence of laparoscopic Roux-Y gastric bypasses globally [1], presumably due to its simple procedure.

Gastrointestinal stromal tumor (GIST) is a very important disease because it is the most frequent and potentially malignant submucosal tumor (SMT). GISTs often originate from the stomach and, as a result of advancements in diagnostic modalities, have recently been detected more frequently. In addition, the incidence of

\section{Springer Open}

(ㅇ The Author(s). 2020 Open Access This article is licensed under a Creative Commons Attribution 4.0 International License, which permits use, sharing, adaptation, distribution and reproduction in any medium or format, as long as you give appropriate credit to the original author(s) and the source, provide a link to the Creative Commons licence, and indicate if changes were made. The images or other third party material in this article are included in the article's Creative Commons licence, unless indicated otherwise in a credit line to the material. If material is not included in the article's Creative Commons licence and your intended use is not permitted by statutory regulation or exceeds the permitted use, you will need to obtain permission directly from the copyright holder. To view a copy of this licence, visit http://creativecommons.org/licenses/by/4.0/. 
GISTs was reported to be more frequent in obese patients undergoing bariatric surgery $(0.8 \%)$ as compared to the general population $(0.001 \%)$ [2]. However, correlation between obesity and GIST is still unknown.

The main treatment for GISTs is complete tumor resection. Local resection is the first choice, but it has been regarded only as the treatment for relatively small or extramural GIST. For large and intramural tumors, extensive surgery such as proximal, distal, and total gastrectomy should be considered depending on the tumor size and location. LSG could also be a concomitant treatment option for both morbid obesity and GIST when the tumor is contained within LSG the excision range. There are, however, few reports about LSG planned for GIST preoperatively.

In this case report, we performed LSG to resect a large GIST located at the gastric fundus in a morbidly obese patient. Here, we report our observation and review the literature on GISTs resected with LSG.

\section{Case presentation}

A morbidly obese 46-year-old Japanese male was diagnosed with a gastric SMT by medical examination and came to our hospital for surgery. The upper gastrointestinal endoscopy and enhanced computed tomography (CT) scan showed an intramural SMT approximately 4 $\mathrm{cm}$ in diameter in the gastric fundus (Fig. 1). A biopsy was performed, and histology showed group 1 at that time.

His body weight (BW) was $105.4 \mathrm{~kg}$, and his body mass index (BMI) was $36.6 \mathrm{~kg} / \mathrm{m}^{2}$. Notably, he had severe visceral adiposity (visceral fat area (VFA) of $386 \mathrm{~cm}^{2}$ ) (Fig. 2a). He also had severe obstructive sleep apnea syndrome (OSAS) (apnea-hypopnea index 79.6) and hypertension. Because of his extreme visceral fat dominated obesity and the size and location of the tumor, we determined that it would be difficult to perform local resection. Instead, we planned LSG as a concomitant treatment for both the tumor and morbid obesity.
After the preoperative examination and 6 months of weight control, the patient lost enough weight (BW 84.2 $\mathrm{kg}$, BMI $29.5 \mathrm{~kg} / \mathrm{m}^{2}$, VFA $305 \mathrm{~cm}^{2}$ ) to undergo LSG safely (Fig. 2b). In the course of this period, although we checked CT scan every 3 months after first visit, no apparent change was detected. The second upper gastrointestinal endoscopy 6 months after the first one, however, revealed that the tumor had increased in size to approximately $5 \mathrm{~cm}$, and that the tumor was diagnosed as a GIST preoperatively based on the histology results.

We performed LSG in our usual manner except that we advanced endoscopy transorally instead of the 37.5-Fr calibration tube along the lesser curvature to observe the tumor intraoperatively. After complete mobilization of the fundus, we observed the intramural tumor located with enough distance from the esophagogastric junction (EGJ) with both laparoscopy and endoscopy (Fig. 3). The stomach was divided using a linear stapler keeping enough distance from the tumor. We contained the specimen including the tumor in a bag and lengthened the $15-\mathrm{mm}$ port incision site by several millimeters. We could retrieve it as usual manner except above relatively easily. The operation time was $156 \mathrm{~min}$ with little blood loss, and the patient was discharged uneventfully on the 7 th postoperative day.

The tumor, measuring at $6.0 \mathrm{~cm} \times 5.0 \mathrm{~cm}$ (Fig. 4), was resected completely and was larger than initial observations during his first visit. Pathological examination revealed that mitotic figures were rarely noted $(1 / 50 \mathrm{HPF})$, and that the tumor cells were strongly positive to both c-kit and CD34 and negative to S-100, desmin, and alpha-smooth muscle actin immunohistochemically. The GIST was diagnosed as low risk in the Miettinen classification and moderate risk in the Modified-Fletcher classification.

In addition, weight loss was also successful as his BMI was $21.0 \mathrm{~kg} / \mathrm{m}^{2}$ at 3 months after the surgery.

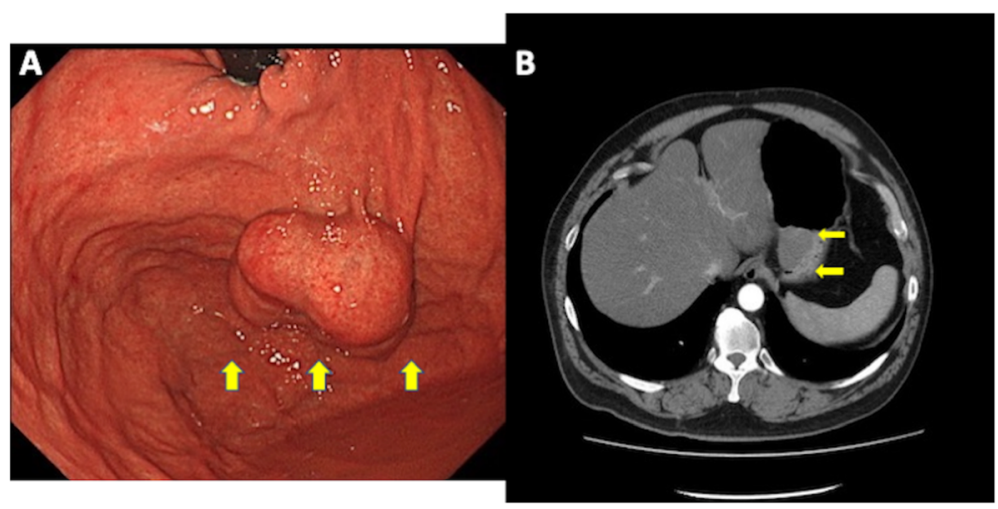

Fig. 1 Preoperative imaging found a large SMT (yellow arrows) at the gastric fundus. a Endoscopy. b CT scan 

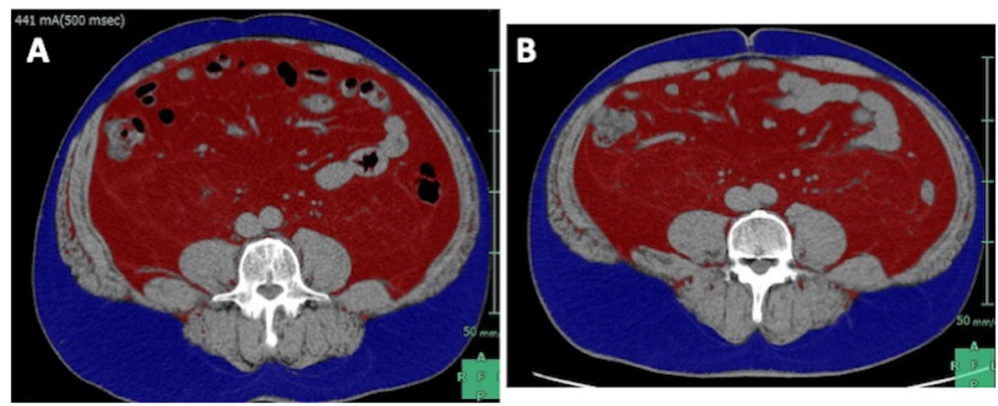

Fig. 2 Assessment of abdominal visceral fat (red) and subcutaneous fat (blue) by Fat Scan. The patient lost enough weight, especially visceral fat. a Before weight loss. b After weight loss

\section{Discussion}

The clinical practice guidelines for GIST in Japan recommend that the indication for laparoscopic surgery must be decided considering the tumor location including whether intra or extramural, the grade of malignancy of the tumor, and the surgeon's experience of laparoscopic surgery, when the tumor is over $5 \mathrm{~cm}$ in size that are associated with an increased risk of rupture [3]. In this case, because the tumor was intramural and not fragile, we considered that there was not a high risk for rupture. Further, the tumor was located at the gastric fundus, which is the deepest part of the abdomen in a patient with extreme visceral fat obesity. A paper about bariatric surgery mentions that open surgery is known to have high risk for complication, and that laparoscopic surgery is safer and recommended for morbidly obese patients [4]. That is why we judged laparoscopic surgery would be safer than open surgery. Moreover, we have enough experience of laparoscopic surgery including LSG at our institution.

We had four surgical options for this patient: local resection, proximal gastrectomy, total gastrectomy, and sleeve gastrectomy. Although the patient was able to lose weight preoperatively, his BMI and visceral fat area remained high. Considering the extreme visceral fat dominated obesity and the location of the tumor, we determined that local resection would not be impossible but be difficult because of the risk of stenosis at the EGJ. We judged that LSG was easier than local resection because we could cut along the longitudinal axis. Moreover, we judged that LSG was the easiest and safest procedure among the three remaining surgical options because it did not need anastomosis. When a tumor is located at the great curvature of the stomach and contained within the excision range, LSG should be considered the best option. In addition, LSG is a concomitant treatment option for both GIST and morbid obesity. In this case, the patient was considered a high-risk surgical candidate because of his extreme visceral fat dominated obesity and obesity-related comorbidity, such as OSAS. Because medical control is very difficult for morbid obesity patients for a long time, the possibility of weight regain would be very high [5] if we did not perform sleeve gastrectomy. For this reason, we performed LSG as a concomitant treatment for obesity management and resection of GIST. Based on endoscopy and CT scans, we expect successful tumor resection with LSG. However, due to the large tumor size and its location near the EGJ, if we determined LSG resection not to be possible intraoperatively, total gastrectomy would be our second option. Total gastrectomy was simpler than proximal gastrectomy in terms of anastomosis.

The patient underwent weight loss for 6 months, which is an average period in our bariatric program,

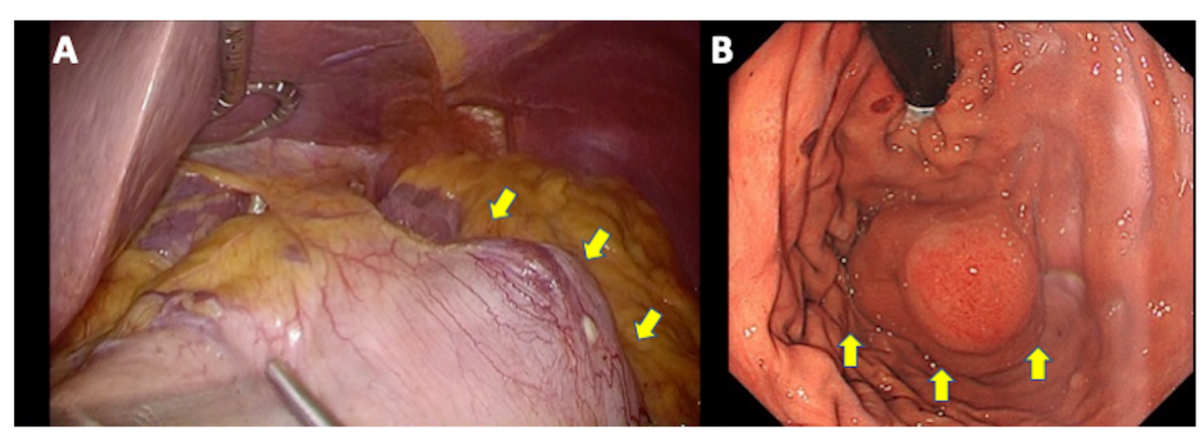

Fig. 3 The tumor (yellow arrows) was observed with a laparoscopy and b endoscopy intraoperatively 


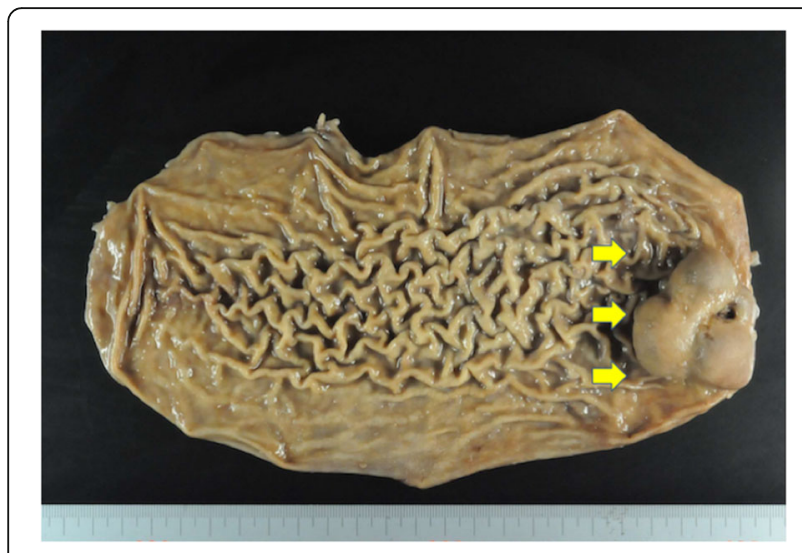

Fig. 4 The resected specimen. The tumor (yellow arrows) was resected completely

because of his extreme visceral fat dominated obesity (VFA of $386 \mathrm{~cm}^{2}$ ). Visceral fat and liver volume are known to be the most important risk factors for upper GI surgery including bariatric surgery for morbidly obese patients. In bariatric surgery, we occasionally experience inoperable cases due to significantly enlarged left lobe of the liver and abundant visceral fat disturbing the surgeon's visualization of upper gastric field. Colles et al. reported that a 5\% weight loss leads to an approximately $10 \%$ reduction in visceral fat volume and over $20 \%$ reduction in liver volume [6]. We judged that preoperative weight loss of at least $10 \%$ would reduce the patient's surgical risk by a sufficient amount even when considering the potential for noncancerous tumor growth while delaying surgical intervention.

The effectiveness of neoadjuvant chemotherapy for GIST has not been established; however, the clinical practice guidelines for GIST in Japan recommend neoadjuvant chemotherapy for selected cases only when the procedure could be diminished [3]. In this case, because the patient was diagnosed with a GIST after the preoperative weight loss period, neoadjuvant chemotherapy was not planned. In addition, if the tumor grew larger or diminished to some extent, we determined that LSG was the best procedure for this case as mentioned above. These were why neoadjuvant chemotherapy was not indicated for this case. As a result, however, the end tumor size during the LSG procedure was larger than anticipated. In retrospect, more frequent checks on the tumor progress should have occurred because GIST is potentially malignant.

A complete literature review was performed , and reports with reference to GISTs resected with LSG are listed in chronological order (Table 1) [7-22]. Although there have been more than 10 papers on GISTs resected with LSG, most reports described small extramural GISTs encountered during operation and resected incidentally $[8-19,21,22]$. We found only two previous reports on LSG planned preoperatively as the treatment for GISTs that were relatively large and intramural [7, 20]. Wang et al. was the first to report on LSG as a GIST treatment [7]. Chetta et al. reported emergency cases in which LSGs were performed for GISTs with acute bleeding [20]. Notably in our case, the size of the tumor was

Table 1 A literature review of GISTs resected with LSG

\begin{tabular}{llllll}
\hline Author & Year & Number of cases & Size of the tumors (cm) & Detection of tumor & Intra/extramural \\
\hline Wang et al. [7] & 2009 & 2 & $2-4.4$ & Pre-op & Intramural \\
Beltran et al. [8] & 2010 & 1 & 1.5 & Incidental & Extramural \\
Yuval et al. [9] & 2014 & 5 & $0.2-2.0$ & Incidental & Extramural \\
Chiappetta et al. [10] & 2015 & 7 & $0.25-1.3$ & Incidental & Extramural \\
Crouthamel et al. [11] & 2015 & 12 & $0.3-2.9$ & Incidental & Extramural \\
Ohanessian et al. [12] & 2016 & 3 & $0.4-0.8$ & Incidental & Extramural \\
Atas et al. [13] & 2016 & 1 & 1.5 & Incidental & Extramural \\
Nickel et al. [14] & 2016 & 1 & 0.9 & Incidental & Extramural \\
Safaan et al. [15] & 2017 & 11 & Incidental & Extramural \\
Viscido et al. [16] & 2017 & 5 & $0.5-1.5$ & Incidental & Extramural \\
Walędziak et al. [17] & 2017 & 14 & $0.3-2.0$ & Incidental & Extramural \\
Saurabh et al. [18] & 2017 & 1 & 1 & Incidental & Extramural \\
Lyros et al. [19] & 2019 & 4 & $0.5-1.1$ & Pre-op & Extramural \\
Chetta et al. [20] & 2019 & 2 & $4.0-4.5$ & Incidental & Intramural \\
Ayoub et al. [21] & 2019 & 1 & 2.0 & Incidental & Pre-op \\
Mendes et al. [22] & 2020 & 11 & $0.2-0.9$ & 6.0 & Extramural \\
Our case & 2020 & 1 & & Extramural & Intramural \\
\hline
\end{tabular}


$6.0 \mathrm{~cm} \times 5.0 \mathrm{~cm}$, which may be the largest GIST resected with LSG planned preoperatively to date.

\section{Conclusion}

We safely performed LSG in a morbidly obese patient with a large gastric GIST. This is the largest GIST concomitantly resected with LSG among previous reports.

\section{Abbreviations}

GIST: Gastrointestinal stromal tumor; LSG: Laparoscopic sleeve gastrectomy; BW: Body weight; BMI: Body mass index; VFA: Visceral fat area; EGJ: Esophagogastric junction; SMT: Submucosal tumor; CT: Computed tomography; OSAS: Obstructive sleep apnea syndrome

\section{Acknowledgements}

We would like to thank Editage (www.editage.com) for English language editing.

\section{Authors' contributions}

$\mathrm{KH}, \mathrm{YS}$, and $\mathrm{KK}$ performed the surgical treatment for this patient. $\mathrm{KH}$ drafted the manuscript. YS and TK critically revised the manuscript. All authors read and approved the manuscript.

\section{Funding}

There is no funding support for this manuscript.

\section{Availability of data and materials}

The datasets generated during the current study are not publicly available because individual privacy could be compromised, but are available from the corresponding author on reasonable request

\section{Ethics approval and consent to participate}

Not applicable.

\section{Consent for publication}

Written informed consent was obtained from the patient for use of medical information and accompanying images for this case report.

\section{Competing interests}

The authors declare that they have no conflict of interest.

Received: 27 May 2020 Accepted: 4 August 2020

Published online: 12 August 2020

\section{References}

1. Welbourn R, Hollyman M, Kinsman R, Dixon J, Liem R, Ottosson J, Ramos A, Våge V, Al-Sabah S, Brown W, Cohen R, Walton P, Himpens J. Bariatric surgery worldwide: baseline demographic description and one-year outcomes from the Fourth IFSO Global Registry Report 2018. Obes Surg. 2019 March;29(3):782-95

2. Sanchez BR, Morton JM, Curet MJ, Alami RS, Safadi BY. Incidental finding of gastrointestinal stromal tumors (GISTs) during laparoscopic gastric bypass. Obes Surg. 2005;15(10):1384-8.

3. Nishida T, Hirota S, Yanagisawa A, Sugino Y, Minami M, Yamamura Y, Otani Y, Shimada Y, Takahashi F, Kubota T. GIST Guideline Subcommittee. Clinical practice guidelines for gastrointestinal stromal tumor (GIST) in Japan: English version. Int J Clin Oncol. 2008;13(5):416-30. https://doi.org/10.1007/ s10147-008-0798-7 Epub 2008 Oct 23.

4. Buchwald $H$, Estok R, Fahrbach $K$, Banel D, Sledge I. Trends in mortality in bariatric surgery: a systematic review and meta-analysis. Surgery. 2007; 142(4):621-32; discussion 632-5. Epub 2007 Jul 18. https://doi.org/10.1016/j. surg.

5. Sjöström L, Narbro K, Sjöström CD, Karason K, Larsson B, Wedel H, Lystig T, Sullivan M, Bouchard C, Carlsson B, Bengtsson C, Dahlgren S, Gummesson A, Jacobson P, Karlsson J, Lindroos AK, Lönroth H, Näslund I, Olbers T, Stenlöf K, Torgerson J, Agren G, Carlsson LM. Effects of bariatric surgery on mortality in Swedish obese subjects. N Engl J Med. 2007;357:741.
6. Colles SL, Dixon JB, Marks P, Strauss BJ, O'Brien PE. Preoperative weight loss with a very-low-energy diet: quantitation of changes in liver and abdominal fat by serial imaging. Am J Clin Nutr. 2006;84(2):304-11.

7. Wang Y, Liu J. Laparoscopic sleeve gastrectomy in the treatment of gastrointestinal stroma tumours in morbid obese patients. BMJ Case Rep. 2009. https://doi.org/10.1136/bcr.06.2008.0204 Epub 2009 May 8.

8. Beltran MA, Pujado B, Méndez PE, Gonzáles FJ, Margulis DI, Contreras MA Cruces KS. Gastric gastrointestinal stromal tumor (GIST) incidentally found and resected during laparoscopic sleeve gastrectomy. Obes Surg. 2010; 20(3):393-6.

9. Yuval JB, Khalaileh A, Abu-Gazala M, Shachar Y, Keidar A, Mintz Y, Nissan A, Elazary R. The true incidence of gastric GIST-a study based on morbidly obese patients undergoing sleeve gastrectomy. Obes Surg. 2014;24(12): 2134-7.

10. Chiappetta S, Theodoridou S, Stier C, Weiner RA. Incidental finding of GIST during obesity surgery. Obes Surg. 2015;25(3):579-83.

11. Crouthamel MR, Kaufman JA, Billing JP, Billing PS, Landerholm RW. Incidental gastric mesenchymal tumors identified during laparoscopic sleeve gastrectomy. Surg Obes Relat Dis. 2015;11(5):1025-8.

12. Ohanessian SE, Rogers AM, Karamchandani DM. Spectrum of gastric histopathologies in severely obese American patients undergoing sleeve gastrectomy. Obes Surg. 2016;26(3):595-602.

13. Atas $H$, Bulus $H$, Akkurt $G$, Yavuz A, Tantoglu U, Alimogullari M, Aydin A. How to approach incidentally detected gastrointestinal stromal tumor during laparoscopic sleeve gastrectomy: a report of two cases. Euroasian Hepatogastroenterol. 2016;6(2):173-5.

14. Nickel F, Probst P, Fischer L. Would you unblind the patient? A case of incidental T1 NO RO GIST in a randomized controlled trial of sleeve gastrectomy versus Roux-en-Y gastric bypass for morbid obesity. Surg Obes Relat Dis. 2016;12(9):e83-5.

15. Safaan T, Bashah M, El Ansari W, Karam M. Histopathological changes in laparoscopic sleeve gastrectomy specimens: prevalence, risk factors, and value of routine histopathologic examination. Obes Surg. 2017;27(7):1741-9.

16. Viscido G, Signorini F, Navarro L, Campazzo M, Saleg P, Gorodner V, Obeide $L$, Moser F. Incidental finding of gastrointestinal stromal tumors during laparoscopic sleeve gastrectomy in obese patients. Obes Surg. 2017;27(8): 2022-5.

17. Walędziak M, Różańska-Walędziak A, Kowalewski PK, Janik MR, Brągoszewski J. Paśnik K. Bariatric surgery and incidental gastrointestinal stromal tumors a single- center study: VSJ Competition, 1st place. Wideochir Inne Tech Maloinwazyjne. 2017;12(3):325-9.

18. Saurabh S. Gastrointestinal stromal tumor: an incidental finding during laparoscopic bariatric surgery. Clin Case Rep. 2017;5(11):1905-6.

19. Lyros O, Moulla Y, Mehdorn M, Schierle K, Sucher R, Dietrich A. Coincidental detection of gastrointestinal stromal tumors during laparoscopic bariatric procedures-data and treatment strategy of a German Reference Center. Obes Surg. 2019;29(6):1858-66.

20. Chetta N, Picciariello A, Nagliati C, Balani A, Martines G. Surgical treatment of gastric GIST with acute bleeding using laparoscopic sleeve gastrectomy: a report of two cases. Clin Case Rep. 2019;7(4):776-81.

21. Ayoub K, Danial AK, Sankari Tarabishi A, Shebli B, Halwani MY, Mahli N. A rare case of incidental finding of GIST during sleeve gastrectomy: case report. Int J Surg Case Rep. 2019;65:161-3. https://doi.org/10.1016/j.ijscr. 2019.10.040 Epub 2019 Nov 1.

22. Mendes JT, Wilson C, Schammel CMG, Scott JD, Schammel DP, Trocha SD. GIST identified during bariatric surgery: to treat or not to treat? Surg Obes Relat Dis. 2020;16(2):282-7. https://doi.org/10.1016/j.soard.2019.10.023 Epub 2019 Nov 18.

\section{Publisher's Note}

Springer Nature remains neutral with regard to jurisdictional claims in published maps and institutional affiliations. 